\title{
Kinetic Energy Corrections for Slip-Stick Behavior in Brittle Adhesives
}

\author{
David J. Macon, Greg L. Anderson
}

\section{Synopsis}

Fracture mechanics is the study of the failure of a body that contains a flaw. In the energy balance approach to fracture mechanics, contributions from the external work and elastic strain energy are accounted for but rarely are corrections for the kinetic energy given. Under slip-stick conditions, part of the external work is expended as kinetic energy. The magnitude of this kinetic energy depends upon the shape of the crack. A specimen with a blunt crack will fail at a high load and the crack will catastrophically travel through the material until the kinetic energy is dissipated. Material with a sharp crack will fail at a lower load but will still be catastrophic in nature.

A kinetic term is incorporated into the energy balance approach. This term accounts for the velocity of the crack after failure and how far the crack travels before arresting. This correction makes the shape of the initiation crack irrelevant. When applied to data generated by tapered double cantilever beam specimens under slip-stick conditions, the scatter in the measured critical strain energy release rate is significantly reduced.

\section{Introduction}

Fracture mechanics is the study of the failure of a body that contains a flaw. Griffith proposed that failure occurs when sufficient energy is released by growth of this flaw to create the new crack surface ${ }^{1}$. The released energy is assumed to come from the elastic or potential energy of the loading system and no other corrections are used. This approach works well for test conditions where the crack propagation is quasi-static and any additional crack growth results from an increase in applied load. This analysis breaks down when the crack propagates in a slip-stick behavior.

This type of crack growth is characterized by a saw-tooth shaped load profile for a tapered double cantilever beam (TDCB) specimen as shown in Figure 1. An initiation load is associated with the profile as the specimen is loaded until the crack instantaneously propagates. As the velocity of the crack decreases and stops, an arrest load is reached. This type of behavior can be repeated several times as the crack moves down the specimen length. The load required for crack initiation is greater than that required for stable crack growth and part of the energy is dissipated as kinetic energy ${ }^{2}$.

Under slip-stick conditions, three features complicate the fracture behavior: inertia forces, rate dependent material behavior, and reflected stress waves. In the case of inertia effects, the crack grows abruptly because a portion of the work that is applied to the specimen is converted to kinetic energy. Rate dependent material behavior is important when considering polymeric materials, because they are typically viscoelastic and very sensitive to temperature and deformation rates. Reflected stress waves are of concern 
because they propagate throughout the material and reflect off the free surfaces. These reflected waves influence the local crack tip stress and strain field that affect the fracture behavior $^{3}$.

Maugis describes the slip-stick behavior in terms of viscoelastic losses or internal friction at the crack tip ${ }^{4}$. His model describes the critical energy release rate, $G$, versus the crack velocity, $v$, as shown in Figure 2. This figure is the superposition of a curve for brittle fracture with dynamic effects, and a curve for viscoelastic losses. He suggests that as long as $G<G_{c}$ (Figure 2 ) the crack propagates at constant velocity at constant $G$. At $G_{c}$ (Point $\mathrm{A}$ ), the crack velocity jumps to the second curve (Point $\mathrm{B}$ ) and the crack propagation is catastrophic. The velocity is too high and the crack slows down to Point $\mathrm{C}$ where the velocity is still too high, then jumps to Point $D$ where the crack suddenly arrests. The velocity increases to $v_{c}$ and jumps to Point B. A slip-stick motion thus occurs.

This approach does a good job describing the slip-stick behavior shown in Figure 1 but does not account for the variability in velocities observed during catastrophic failure. In certain experimental conditions, the magnitude of the initiation load can vary significantly with concomitant variation in the crack velocities. Several studies have examined the relationship between the crack driving force and the crack velocity ${ }^{5-8}$. The magnitude of the crack driving force and hence the crack velocity strongly depends upon how the sample initiation crack is started.

Initiating a proper crack can be very difficult. Marshall et al, found that for polystyrene the critical stress intensity factor (evaluated at crack instability), which should be a constant, independent of test method, and specimen geometry, varied over a wide range ${ }^{9}$. This was shown to be dependent upon the method of notching employed. This behavior was also observed by Hine et al, who were able to reduce the variability in slip-stick behavior by reducing the probability of a region of plastic deformation occurring in front of the starter crack ${ }^{10}$. Dillard et al, describe the importance and difficulty in generating an appropriate initial flaw ${ }^{11}$. There is some uncertainty as to whether the initiation crack is ever adequate to give an accurate measure of the resistance of the material to fracture during slip-stick behavior

In this work, we use the TDCB geometry to measure the critical energy release rate for an adhesive that exhibits slip-stick behavior. A kinetic energy term is included in the energy balance approach. The resulting expression accounts for the variability introduced by the different types of initiation cracks.

\section{Experimental}

\section{Materials}

The epoxy resin used in this study was TIGA $321^{\circledR}$ manufactured by Resin Technology Group (RTG). The curing agent used in the TIGA $321^{\circledR}$ is a mixture of amines. The adhesive contains both Kevlar ${ }^{\circledR}$ fibers and milled glass fibers. 


\section{Preparation of TDCB Specimens}

The epoxy was vacuum mixed and then coated onto a D6AC steel TDCB adherend that conformed to ASTM D 3433. A schematic of the specimen is shown in Figure 3. Excess adhesive was applied to the beam and allowed to squeeze out as the two adherends were pressed together. The extruded adhesive was removed from the side of the beams prior to curing. The bondline thickness was set at $0.127 \mathrm{~cm}$ using Teflon ${ }^{\circledR}$ spacers controlled to a tolerance of $0.0127 \mathrm{~cm}$. After coating, the specimens were placed in a jig that would maintain alignment and a slight compressive load was applied. The samples were cured at $40.5^{\circ} \mathrm{C}$ for 48 hours and then rapidly cooled to $22.2^{\circ} \mathrm{C}$. This cure cycle was chosen because it duplicates the cure schedule of the adhesive as it is used on one of the products produced by the Thiokol Propulsion Group of Alliant Techsystems.

\section{Critical Strain Energy Measurements}

Testing was conducted using a screw-driven testing machine (e.g., Instron ${ }^{\circledR}$ or Satec ${ }^{\circledR}$ ) outfitted with a temperature-control chamber. The tests were most often performed in displacement control at a crosshead speed of $0.0127 \mathrm{~cm} /$ minute. Unless otherwise specified, all tests were performed at $22.2^{\circ} \mathrm{C}$. At this test temperature and deformation rate, TIGA $321^{\circledast}$ displayed slip-stick behavior.

A variety of techniques were used to generate flaws in the adhesive bondline. Among these were embedding a razor in the adhesive bondline during fabrication, tapping a razor blade into the adhesive bondline at cold temperature, fatigue loading the specimen at cold temperature, or loading the beam and exposing the bondline front to liquid nitrogen. Most of these approaches introduced a visibly sharp crack into the bondline (with the embedded razor blade being a questionable exception). However upon loading the specimens at the specified temperature and deformation rate, a load would be reached at which the crack would propagate down the entire length of the beam. The specimens with the embedded razor bladed also reached a critical load but the crack would only propagate a short distance, allowing more than one loading peak before failing catastrophically. Because of this, the embedded razor was selected as the method for inducing a flaw into the bondline.

The crack velocity was measured using crack propagation gauges manufacture by Micromeasurements (part number TK09-CPC03-003/DP). The gauges consist of parallel electrical circuits that produce a change in voltage, as the circuits are broken. These gauges are $3.86 \mathrm{~cm}$ in length, which is shorter than the full length of the adhesive bondline, but this will be addressed later in the results and discussion section. Measurements were taken from the gauge at a rate of 222,000 readings/s.

Measurement of the Acoustic Velocity in Cured TIGA $321^{\circledR}$

The measurement of the acoustic velocity in the adhesive material was done using a pulse multiple echo technique, which can simultaneously measure the acoustic wave-speed and 
the thickness of the sample. A pulsed ultrasound wave from a $1 \mathrm{MHz}$ (center frequency) transducer was excited by a HV pulsed from a Ritec SP-801 pulse to the transducer. The beam was directed normally through one of the ends of the adhesive sample and echoes from the front surface, back surface, and the surface of another passive transducer located behind the sample were reflected back to the active transducer. This is shown in Figure 4. A Ritec RDX-6 Diplexer was used to separate the transmitted pulse signal from the transducer signal produced by the reflected energy. A Ritec B40 Broadband Receiver amplified the electric signals generated by the reflections striking the transducer. The amplified signal was digitized by a Lecroy LC9314L digital oscilloscope and stored in a computer file for later reference and analysis.

The theory behind the technique is explained as follows. By examining Figure 4, it can be seen that the difference between the arrival times of the reflector reflected waves with and without the sample in the water medium are

$$
t_{r o}-t_{r s}=\frac{2 d}{v_{1}}-\frac{2 d}{v_{2}}
$$

where $t_{r o}$ is the arrival time of the reflector reflected waves without the sample, $t_{r s}$ is the arrival time of the reflector reflected waves with the sample, $d$ is the thickness of the sample, $v_{1}$ is the acoustic velocity in water, and $v_{2}$ is the acoustic velocity in the cured adhesive.

The difference in arrival times of the front surface and back surface reflections are

$$
t_{b}-t_{f}=\frac{2 d}{v_{2}}
$$

where $t_{f}$ is the arrival time from the sample front surface and $t_{b}$ is the arrival time from sample back surface.

Thus,

$$
2 d=\left(t_{r o}-t_{r s}+t_{b}-t_{f}\right) \nu_{1}
$$

and,

$$
v_{2}=\frac{\left(t_{r o}-t_{r s}+t_{b}-t_{f}\right)}{t_{b}-t_{f}} v_{1}
$$




\section{Results and Discussion}

\section{Slip-Stick Fracture Behavior}

The dependence of the critical strain energy release rate for TIGA $321^{\circledR}$ upon temperature and deformation rate is shown in Table 1 . Included in the table is the coefficient of variation $(\mathrm{CV})$, which is the standard deviation, divided by the mean average. The reported values are evaluated at crack instability using traditional relations for calculating the strain energy release rate for a TDCB specimen as discussed in ASTM D 3433. Inspection of the table shows that the material exhibits slip-stick behavior at cold temperatures and high deformation rates as expected. Of particular concern is the considerable data scatter under these test conditions. These fracture energy values can be used to establish safety factors, and if a statistical penalty is applied to the mean average (e.g., 3-sigma), the resulting values will be extremely low. Another problem with basing the fracture energy on measurements taken at crack instability is that a portion of the calculated energy is kinetic energy. Also, the failure load has a strong dependence upon crack shape.

If the slip-stick behavior is closely examined, certain observations can be made. For test conditions of $22.2^{\circ} \mathrm{C}$ and $0.0127 \mathrm{~cm} /$ minute, two load peaks are observed. A typical load profile is shown in Figure 5. The sample is loaded until a critical value is reached. The crack then propagates down the length of the beam in a catastrophic manner decreasing in velocity until the crack halts. The beam then continues to load under the constant applied displacement rate until a new critical load is reached and the crack propagates down the remaining length of the beam in a catastrophic manner. If the load of the first failure peak is plotted versus crack length obtained in the first unstable crack growth event as shown in Figure 6, a linear relationship is observed. The more interesting result is observed when the second peak is plotted versus crack length from the first catastrophic failure event as shown in Figure 6. There is again a linear relationship between the load and the crack length, but it behaves in an opposite manner to that of the first load peak. Generally, the higher the load is on the first peak, the lower the second peak will be. This phenomenon can be repeated by unloading the sample after reaching the first peak, staging the specimen for 3 days under ambient conditions, and then reloading. Even after the staging period, the second peak follows the same behavior indicating that this behavior is associated with the adhesive and not the loading setup.

These types of trends have been previously observed in the literature. Hine et al, observed that the crack velocity was proportional to the crack length and also to the initiation load ${ }^{10}$. This is consistent with our observations. For dissipative processes that involve an increase in the energy release rate associated with crack growth, the material is said to exhibit R-curve behavior. The lack of an R-curve indicates that fracture is only associated with the intrinsic toughness of the material and no other toughening mechanism is involved ${ }^{12}$. Du et al, observed that the intrinsic toughness for their modified epoxy decreases with increasing crack velocity ${ }^{13}$. Generally, there is an inverse relationship between the intrinsic toughness and crack velocity ${ }^{3}$. 
The implications of this inverse relationship are a bit disturbing. As discussed previously, the load at failure depends upon how the specimen is precracked, and the general consensus is that a sharp initial crack is ideal. Cracks generated by slip-stick behavior are extremely sharp and yet subsequent loading gives considerable scatter in the failure loads. Because of this, there is always an associated uncertainty as to whether the crack is generated under the proper conditions to give an accurate measure of the critical strain energy release rate.

\section{Kinetic Energy Contribution to the Critical Strain Energy Release Rate}

The crack shape will affect the crack velocity during catastrophic failure. A sharp crack will fail at lower loads with the result of a slower crack velocity. The opposite trend in velocity will be followed for blunt cracks. If the behavior of the velocity can be normalized to some standard, the effect of the crack shape becomes irrelevant. This correction is found by calculating the kinetic energy expended during catastrophic failure. In the energy balance approach to fracture mechanics, quasi-static crack propagation is described as the conversion of the work done by the external force and the available elastic energy stored in the bulk of the specimen into surface free energy. If the crack is not quasistatic but propagates unstably in a slip stick faction, a kinetic energy term is required in the energy balance. For work on a system, the total energy balance for external work, $W$, is given by

$$
W=U+T+D
$$

where $U$ is the elastic strain energy, $T$ is the kinetic energy, and $D$ is the dissipative energy associated with fracture.

For a body with a given cross-sectional area, $A$, the condition for crack growth is

$$
\frac{\partial D}{\partial A}=\frac{\partial(W-U-T)}{\partial A}
$$

The term on the left hand of the expression is called the critical strain energy release rate, $G_{c}$, and is a measure of the energy required to propagate a crack over some unit area. It is assumed that the body is of uniform thickness, $b$, in which case equation (1) becomes

$$
G_{r}=\frac{1}{b} \frac{\partial(W-U-T)}{\partial a}
$$

Yaniv et al., derived the critical strain energy release rate for a specially configured $\mathrm{TDCB}^{14}$. A similar derivation will be presented here for a differently configured TDCB. The kinetic energy for a beam is given by the Bernoulli-Euler beam theorem as 


$$
T=\frac{1}{2} \int_{0}^{a} \rho A(x)\left[\frac{\partial y(x, t)}{\partial t}\right]^{2} d x
$$

where $A(x)$ is the cross-sectional area, $\rho$ is the material density, $a$ is the current length of the crack, $y$ is the beam deflection, and $x$ is the location along the beam length.

The transverse velocity can be written by the chain rule as

$$
\frac{\partial y}{\partial t}=\frac{\partial y}{\partial a} \frac{\partial a}{\partial t}+\frac{\partial y}{\partial x} \frac{\partial x}{\partial t}=\frac{\partial y}{\partial a} \dot{a}
$$

Mostovoy et al., gives the displacement for a single cantilever beam as ${ }^{15}$

$$
y=\frac{12 P}{E b} \int_{0}^{a} \frac{x^{2}}{h^{3}} d x+\frac{6(1+v)}{E b} \int_{0}^{a} \frac{1}{h} d x
$$

where $P$ is the applied load, $E$ is Young's modulus of the beam, $h$ is the height of the beam, and $v$ is Poisson's ratio of the beam.

Differentiating the displacement with respect to crack length and setting $v=1 / 3$ gives

$$
\frac{\partial y}{\partial a}=\frac{4 P}{E b}\left[\frac{3 a^{2}}{h^{3}}+\frac{1}{h}\right]=\frac{4 P}{E b} m
$$

For a TDCB, the term is brackets is assigned to a constant, $m$, and is given the value $35.43 \mathrm{~cm}^{-1}$. In the actual fabrication of the TDCB, the adhesive bondline doesn't start at the loading point but rather where the tapering of the beam begins as shown in Figure 7. For the bondline area, the height as a function of $x$ is given as

$$
\frac{3 x^{2}}{h^{3}}+\frac{1}{h}=m
$$

The real root of this expression in terms of $h$ can be solved. However, the solution is clumsy to work with numerically but can easily be fit with a power law in the form

$$
h=0.445 x^{0.663}
$$

A comparison of the fit between this power law and the numerical solution to the roots of $m$ is shown in Figure 8. It can be seen that there is an excellent agreement between the two curves.

For our experimental conditions, the load, $P$, given in equation (5) will not be constant but will vary from an initiation value, $P_{l}$, to an arrest value, $P_{A}$. It is assumed that the 
load profile will be linear as the crack propagates. Thus, the load as a function of $x$ is given the form

$$
P(x)=P_{1}-\left(P_{1}-P_{A}\right) \frac{x}{a}
$$

The crack velocity, $\dot{a}$, is also assumed to be a linear function ranging from an initial velocity, $\dot{a}_{o}$, at crack initiation and decreasing to zero at crack arrest. The velocity as a function of $x$ is assumed to be in the form

$$
\dot{a}(x)=\frac{\dot{a}_{o}(a-x)}{a}
$$

Substituting equations (6)-(8) into equation (2), integrating with respect to $x$, and then differentiating with respect to $a$ yields

$$
\frac{1}{b} \frac{\partial T}{\partial a}=0.331 \frac{\rho m^{2} \dot{a}_{o}^{2}}{E^{2} b^{2}}\left[P_{l}^{2}+0.831 P_{l} P_{A}+0.369 P_{A}^{2}\right] a^{0.663}
$$

In equation (1), the contribution to the critical strain energy release rate from the work and elastic strain energy is given $b^{2}$

$$
\frac{1}{b} \frac{\partial(W-U)}{\partial a}=\frac{P^{2}}{2 b} \frac{\partial C}{\partial a}
$$

where $C$ is the compliance of the beam. Mostovy et al., gives for a TDCB with a constant $m^{15}$

$$
\frac{1}{b} \frac{\partial(W-U)}{\partial a}=\frac{P^{2}}{2 b} \frac{8}{E b} m
$$

The critical energy release rate for slip-stick behavior in the TDCB can be found by subtracting equation (9) from equation (11).

A plot of the $\frac{\partial(W-U-T)}{\partial a} / \frac{\partial(W-U)}{\partial a}$ versus crack velocity is shown in Figure 9. In generating this plot, $P_{l}=4003 \mathrm{~N}, P_{l}=1334 \mathrm{~N}, b=2.54 \mathrm{~cm}, E=200 \mathrm{GPa}, a=7.62$ $\mathrm{cm}, \rho=7.84 \mathrm{~g} / \mathrm{cm}^{3}$. Inspection of the figure shows that the contribution from the kinetic energy remains insignificant until relatively large velocities are reached (e.g., 101.6 $\mathrm{m} / \mathrm{sec}$ ). Once these velocities are reached, the kinetic contribution becomes more significant.

Equation (9) requires the velocity and crack length to be known. Under certain test conditions for TIGA $321{ }^{\circledR}$ bonded to a TDCB specimen, the crack will propagate 
catastrophically down the length of the beam. These are considered no tests. Only test conditions that generated two load peaks are used.

The crack velocity was measured using crack propagation gauges. A typical fit of the change in voltage with time is shown in Figure 10. This profile was consistent for all specimens tested. The crack velocity is linear until about half the gauge is traveled then the crack starts to accelerate. This phenomenon is unrelated to the kinetic energy generated during the failure event. Instead it is believed that this is caused by the crack meeting reflected waves. These waves are generated during the failure event and travel down the length of the beam until a free surface is reached. The reflected waves return to the point of initiation and collide with the advancing crack tip.

This assumption seems reasonable if the speed of sound for TIGA $321^{\circledR}$ is compared to the linear component of the crack velocity. Using the acoustic emission technique described in the experimental section, the speed of sound for TIGA $321^{(1)}$ was measured at $2794 \mathrm{~m} / \mathrm{s}$. The time it takes for the generated wave at the measured velocity to travel to the end and back before it encounters the advancing crack was calculated. The difference between the calculated and experimental time was on average $4 \%$. This appears to be a reasonable explanation for the observed behavior but it is realized that the complete picture of the underlying mechanisms will be more complex.

The data from a set of TDCB specimens tested at $22.2^{\circ} \mathrm{C}$ and $0.0127 \mathrm{~cm} /$ minute is given in Table 2. Included in the table is the initiation load, the arrest load, initial crack velocity, and crack length. There is considerable scatter in the initiation load, which indicates the variation in crack shape at initiation. The arrest load value has little scatter and is generally accepted to be a material property. It has been observed previously that the crack will arrest when the energy release rate falls approximately to that of the epoxy matrix $^{16}$. However when reflected stress waves are significant, the dynamic arrest toughness of the material will be less than the true material resistance ${ }^{17}$. Because of this, care must be taken in assigning a material property to the arrest load. The reported initial velocity and crack length are generally a linear function of initiation load.

The critical strain energy release rates obtained with and without removing the kinetic energy term are shown in Figure 11. The energy release rate with the kinetic energy is given by equation (11). The kinetic energy is corrected for by subtracting equation (9) from equation (11). The following values were used: $b=2.54 \mathrm{~cm}, E=200 \mathrm{GPa}$, $\rho=7.84 \mathrm{~cm} / \mathrm{in}^{3}$. The figure shows that accounting for the kinetic energy reduces the scatter in the data and gives a more accurate result for the material property. The scatter changes from a coefficient of variation of $22.8 \%$ for the uncorrected calculation to $9.6 \%$ for the corrected one. While the reduction in scatter is substantial if does not eliminate it. It is likely that a significant portion of the remaining scatter is caused by the assumption of a constant crack velocity. Figure 10 shows the crack beginning to accelerate after crack initiation. As mentioned previously, this non-linearity was mostly likely cause by reflected waves. Because of this, the crack will travel a different distance that it would if there were no reflected waves. Unfortunately, the reflected waves are an inherent part of 
the TDCB specimen. Even with this limitation, accounting for the kinetic energy gives significant improvements in accuracy.

\section{Summary}

The general conclusion drawn from this study is that a significant portion of the scatter in the strain energy measured under slip-stick conditions is a result of kinetic energy. The magnitude of this kinetic energy contribution depends upon the shape of the crack. A blunt crack will cause the TDCB specimen to fail at high loads with a significant portion of that energy dissipated as unstable crack propagation. Sharper cracks will also cause unstable failure, but the velocity of the crack will be smaller in magnitude than for the blunt crack.

A correction to the energy balance approach that accounted for contributions from the kinetic energy was presented. Using this correction, the scatter in the critical stain energy release rate was significantly reduced. Also, the shape of the starter crack was irrelevant when the correction factor was used.

\section{Acknowledgements}

The authors wish to express their gratitude to Mr. M. L. Mclennan and Dr. S. I. Harper for their assistance and advice during the course of these studies. 
1. Griffith, A. A., Phil. Trans. Roy. Soc., A221, 163 (1920).

2. Kinlock, A. J., Adhesion and Adhesives: Science and Technology, Chapman and Hall, London, 1987.

3. Anderson, T. L., Fracture Mechanics: Fundamentals and Applications, CRC Press, Boca Raton, 1991.

4. Maugis, P., J. Mat. Sci., 20, 3041 (1985).

5. Schroedar, J. A., J. Mat. Sci, 23, 3037 (1988).

6. Andrews, E. H., and A. J. Stevenson, J. Mat. Sci., 13, 1680 (1978).

7. Atkins, A. G., Lee, C. S., and R. M. Caddell, J. Mat. Sci., 10, 1381 (1975).

8. Takemori, M. T., Morelli, T. A., and J. McGuire, J. Mat. Sci., 24, 2221 (1989).

9. Marshall, G. P, Culver, L.E., and J. G., Williams, Int. J. Fract., 9, 295 (1973).

10. Hine, P. J., Duckett, R. A., and I. M. Ward, J. Mat. Sci., 19, 3796 (1984).

11. Dillard, D. A., McDaniels, P. R., and J. A. Hinkley, J. Mat. Sci. Let., 12, 1258 (1993).

12. Du., J., Thouless, M. D., and A. F., Yee Int. J. Fract., 92, 27 (1998).

13. Du, J., Thouless, M. D., and A. F. Yee, Acta Mater., 48, 3581 (2000).

14. Yaniv, G., and I. M. Daniel, ASTM STP 972, American Society for Testing and Materials, Philadelphia, 1988, 241.

15. Mostovoy, S., Crosley, P. B., and E. J. Ripling, J. Mat., 2, 661 (1967).

16. Tvergaard, V., and J. W. Hutchinson, J. Mech. Phys. Solids, 40, 1377 (1992).

17. Kalthoff, J. F., Beinent, J., and S. Winkler, ASTM STP 627, American Society for Testing and Materials, Philadelphia, 1988, 241. 
Table 1 - Critical strain energy results for TIGA $321^{\circledR}$. Results shown are the mean average and the coefficient of variation. Four samples are tested per condition.

\begin{tabular}{|c|c|c|c|}
\hline $\begin{array}{c}\text { Deformation Rate } \\
(\mathrm{cm} / \mathrm{minute})\end{array}$ & Test Temperature $\left({ }^{\circ} \mathrm{C}\right)$ & $\begin{array}{c}\mathrm{GI}_{\mathrm{c}}\left(\mathrm{J} / \mathrm{m}^{2}\right) \\
\text { Average }(\mathrm{CV})\end{array}$ & Failure Type \\
\hline 1.27 & \multirow{4}{*}{-6.7} & $1273(18.5)$ & Slip-Stick \\
\hline 0.127 & & $1394(45.1)$ & Slip-Stick \\
\hline 0.0127 & & $1931(10.1)$ & Slip-Stick \\
\hline 0.00127 & & $2326(16.4)$ & Slip-Stick \\
\hline 1.27 & \multirow{4}{*}{4.4} & $1779(29.8)$ & Slip-Stick \\
\hline 0.127 & & $2304(44.4)$ & Slip-Stick \\
\hline 0.0127 & & $2646(37.4)$ & Slip-Stick \\
\hline 0.00127 & & $3366(17.4)$ & Slip-Stick \\
\hline 1.27 & \multirow{4}{*}{22.2} & $4789(9.3)$ & Slip-Stick \\
\hline 0.127 & & $5091(9.4)$ & Slip-Stick \\
\hline 0.0127 & & $5626(7.2)$ & Stable \\
\hline 0.00127 & & $5225(8.9)$ & Stable \\
\hline 1.27 & \multirow{4}{*}{32.2} & $6171(2.5)$ & Stable \\
\hline 0.127 & & $5964(3.0)$ & Stable \\
\hline 0.0127 & & $5861(4.9)$ & Stable \\
\hline 0.00127 & & $5792(3.5)$ & Stable \\
\hline 1.27 & \multirow{4}{*}{46.1} & $6486(5.1)$ & Stable \\
\hline 0.127 & & $7033(2.8)$ & Stable \\
\hline 0.0127 & & $6311(6.2)$ & Stable \\
\hline 0.00127 & & $4505(5.2)$ & Stable \\
\hline
\end{tabular}


Table 2 - Data from TDCB Specimen Testing

\begin{tabular}{|c|c|c|c|}
\hline $\begin{array}{c}\text { Initiation Load } \\
(\mathrm{N})\end{array}$ & $\begin{array}{c}\text { Arrest Load } \\
(\mathrm{N})\end{array}$ & $\begin{array}{c}\text { Initial Crack Velocity } \\
(\mathrm{m} / \mathrm{s})\end{array}$ & $\begin{array}{c}\text { Crack Length } \\
\mathrm{cm}\end{array}$ \\
\hline 2740 & 1477 & 116 & 4.3 \\
\hline 3465 & 1446 & 183 & 6.6 \\
\hline 3220 & 1481 & 163 & 5.3 \\
\hline 3727 & 1419 & 204 & 8.1 \\
\hline 4039 & 1361 & 229 & 9.6 \\
\hline 2758 & 1521 & 120 & 3.8 \\
\hline 3683 & 1477 & 200 & 8.9 \\
\hline 3505 & 14777 & 185 & 9.1 \\
\hline 6269 & 1468 & 164 & 7.4 \\
\hline 3065 & 1530 & 146 & 6.1 \\
\hline 3487 & 1508 & 183 & 7.9 \\
\hline 3692 & 1446 & 201 & 9.1 \\
\hline 3432 & 1334 & 222 & 11.2 \\
\hline 3821 & 1419 & 212 & 9.9 \\
\hline 2896 & 1570 & 132 & 5.8 \\
\hline 2740 & 1477 & 115 & 4.3 \\
\hline
\end{tabular}




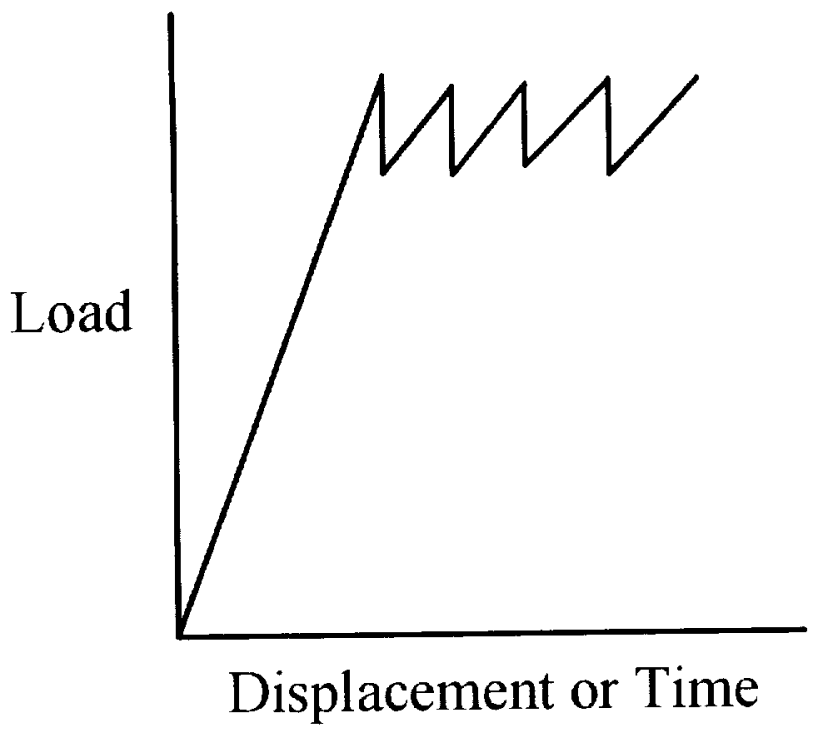

Figure 1: Slip-stick load profile for a TDCB specimen. 


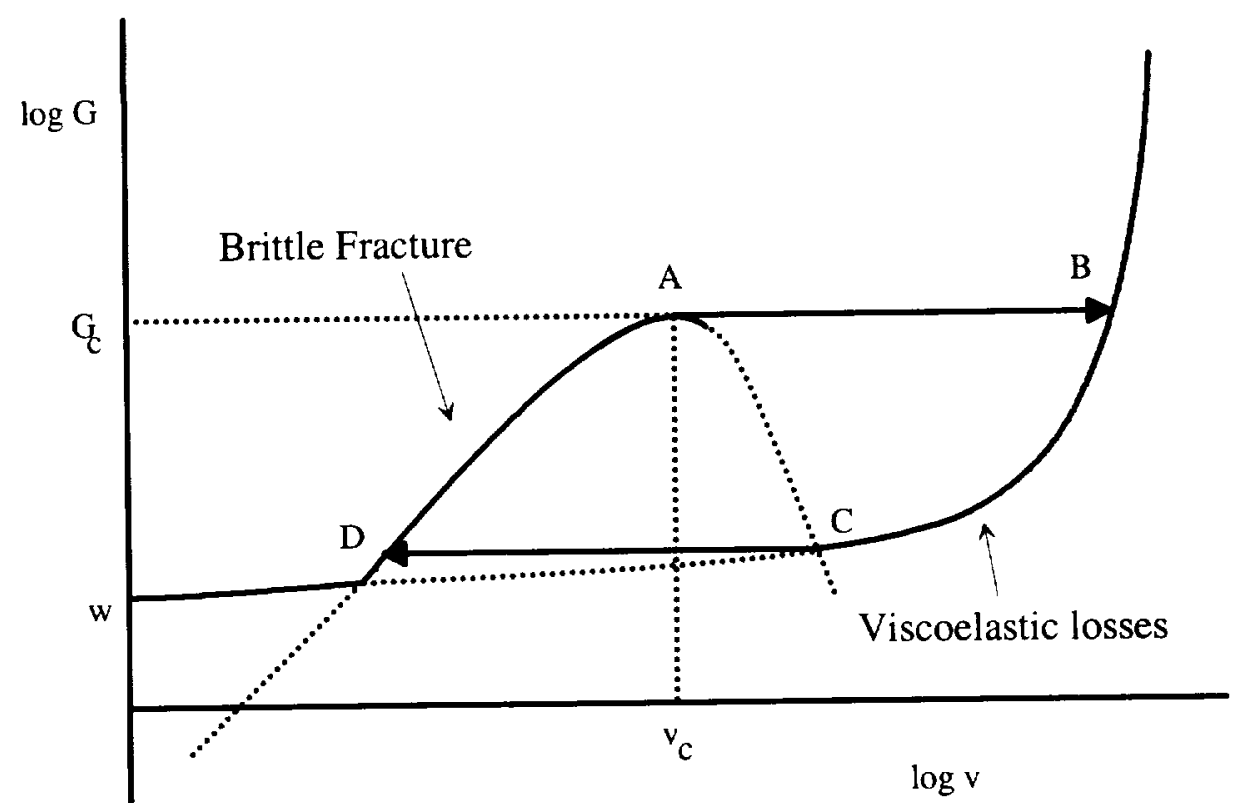

Figure 2: Theory proposed by Maugis to explain slip-stick behavior. 


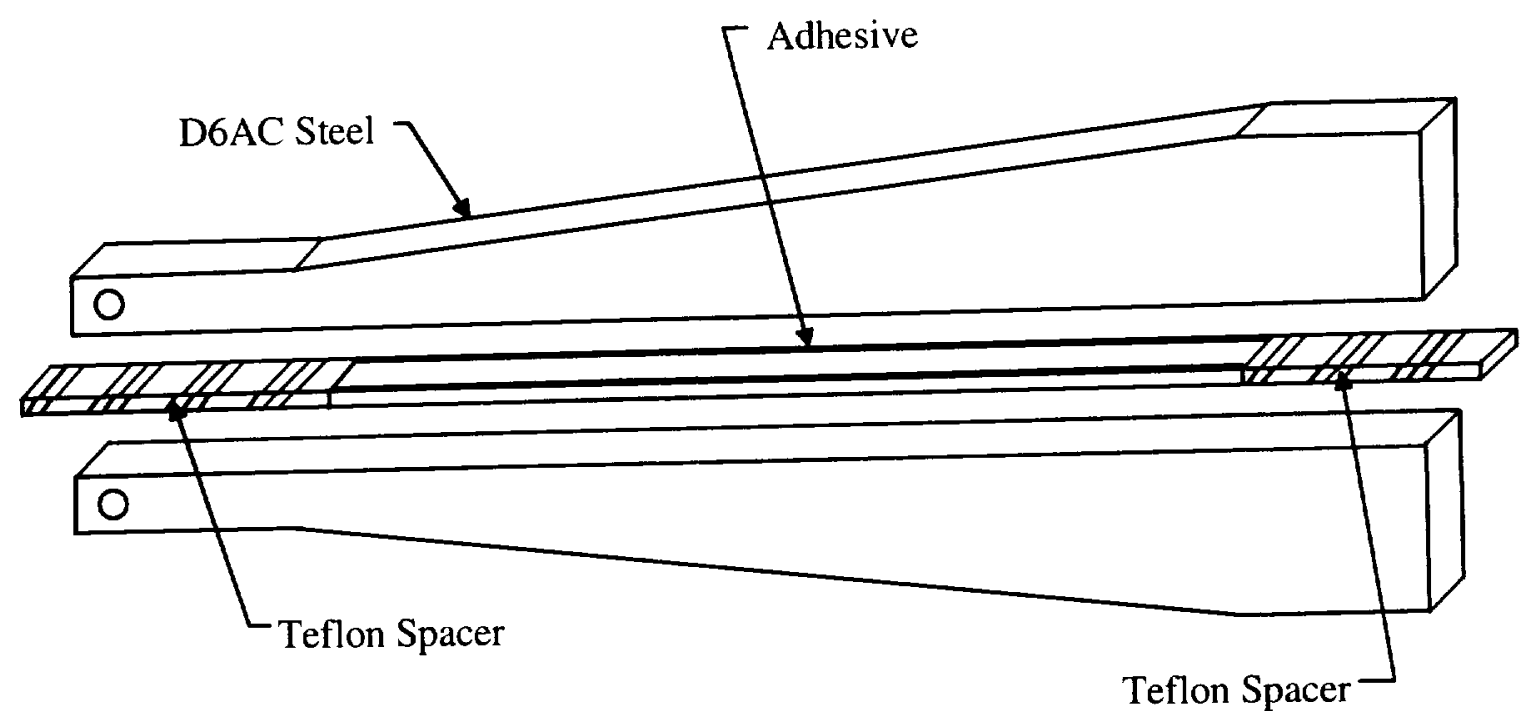

Figure 3: Bonding setup for TDCB specimen. 


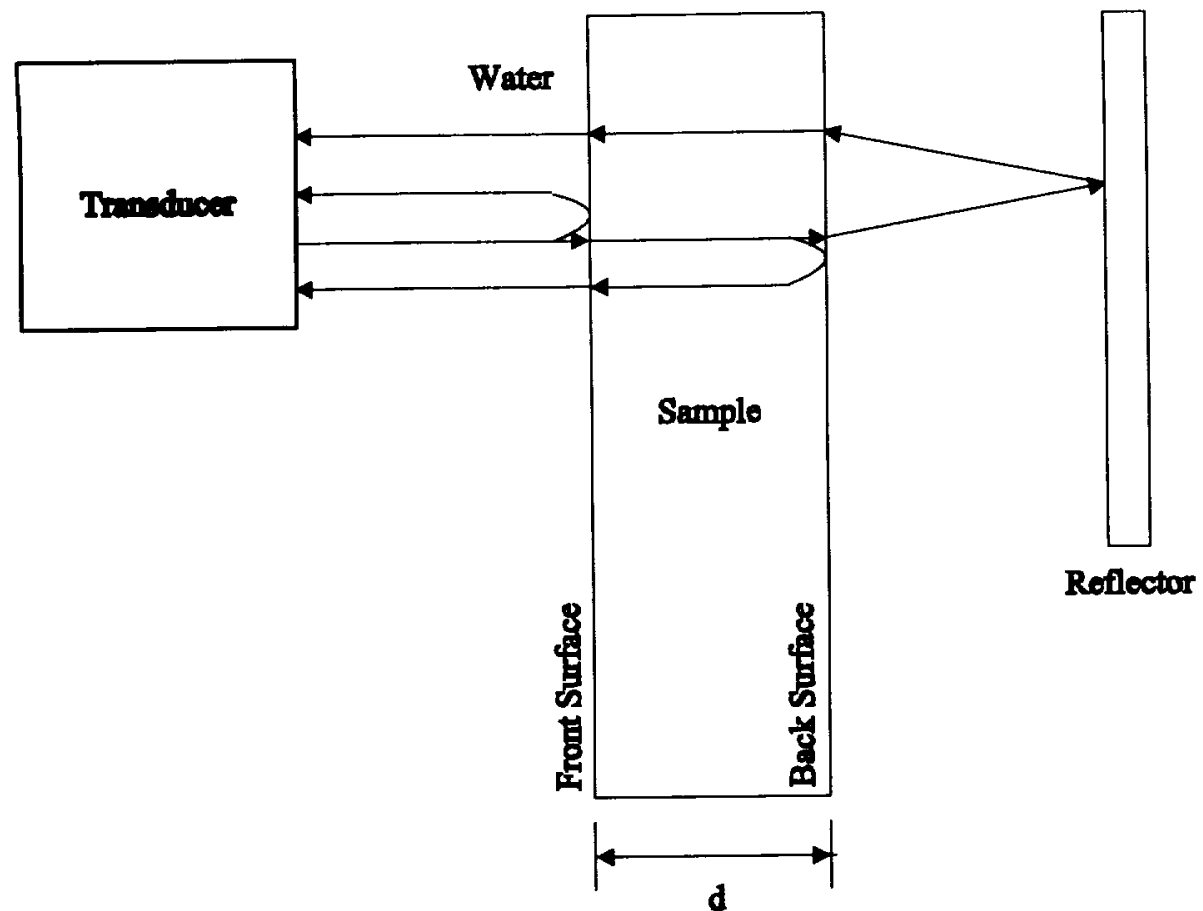

Figure 4: Diagram of apparatus used to measure velocity of sound in a material. 


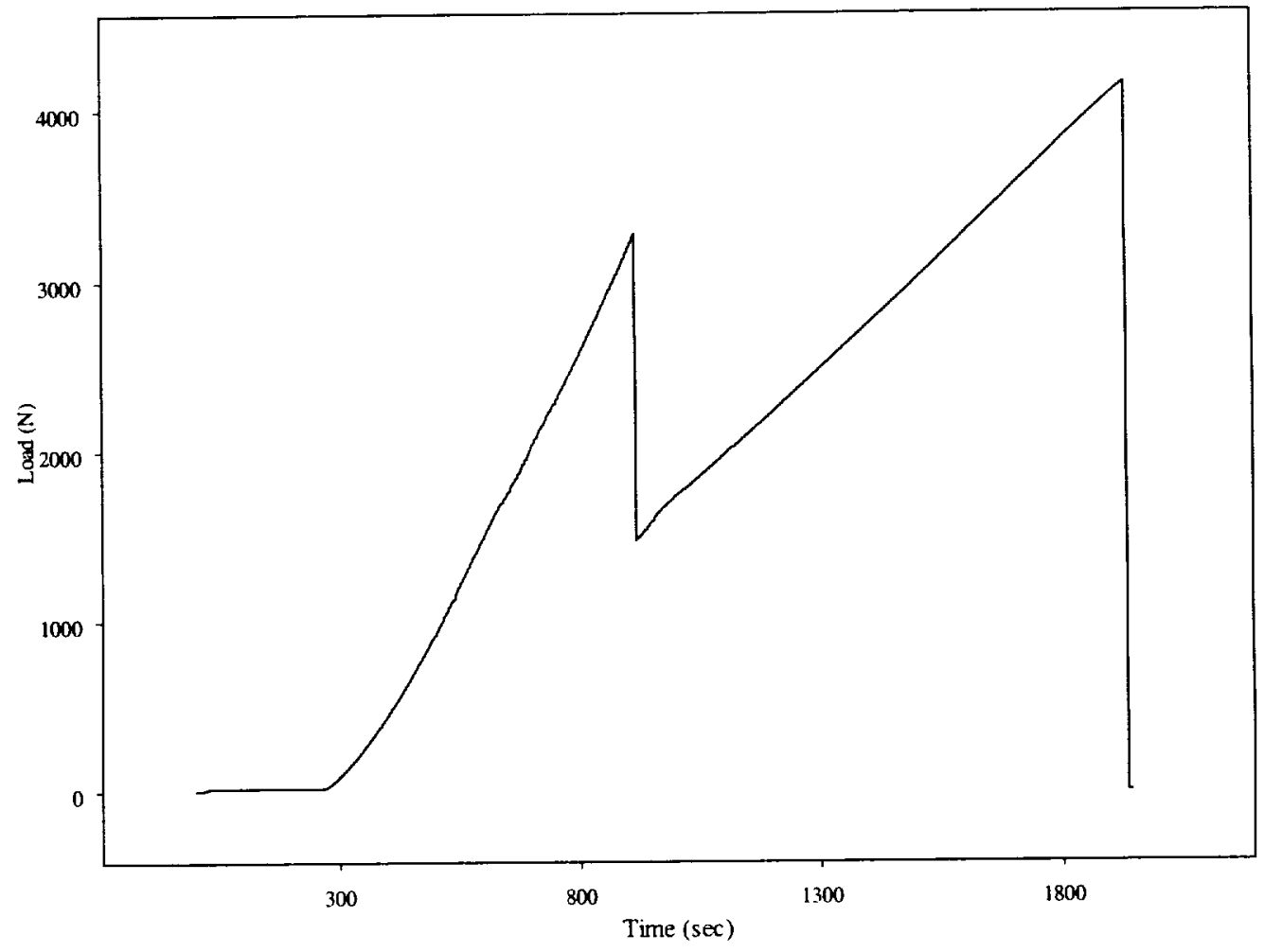

Figure 5: Typical load profile for TIGA $321^{\circledR}$ loaded at $0.0127 \mathrm{~cm} / \mathrm{min}$ at $22.2^{\circ} \mathrm{C}$. 


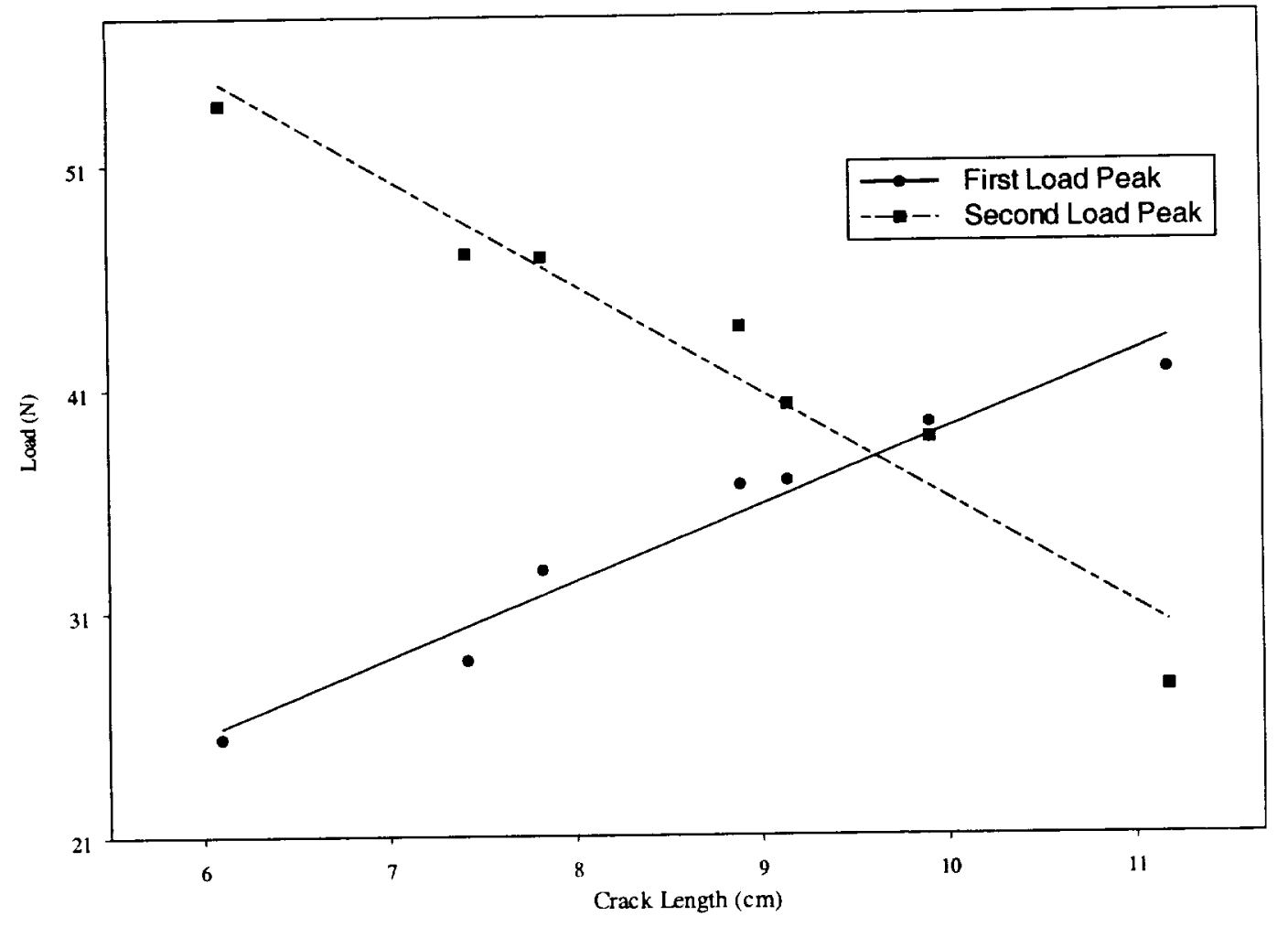

Figure 6: First and second load peaks versus crack length for TIGA $321^{\otimes}$ loaded at $0.0127 \mathrm{~cm} / \mathrm{min}$ at $22.2^{\circ} \mathrm{F}$. 


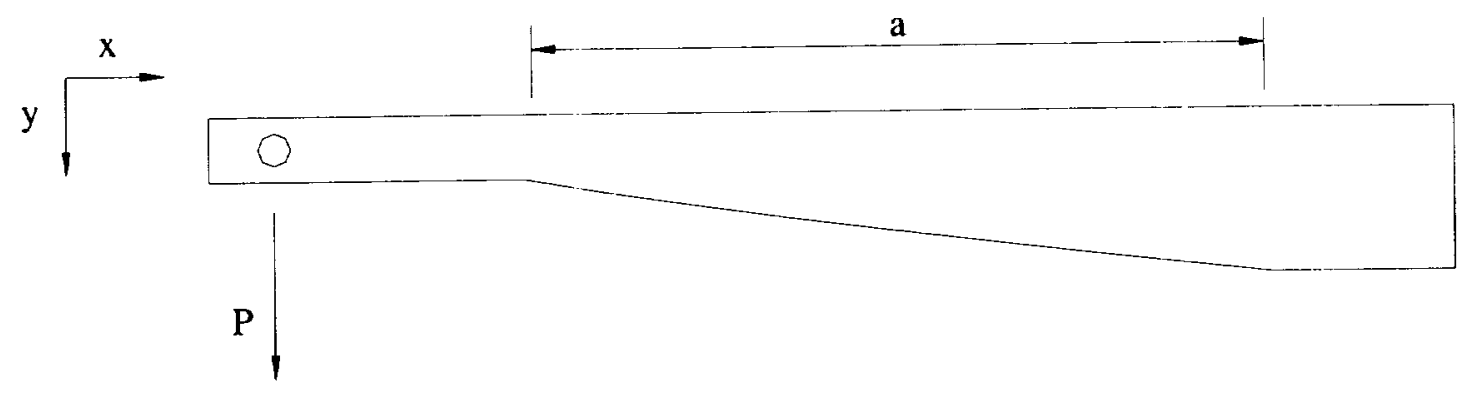

Figure 7: Loading schematic for TDCB specimen. 


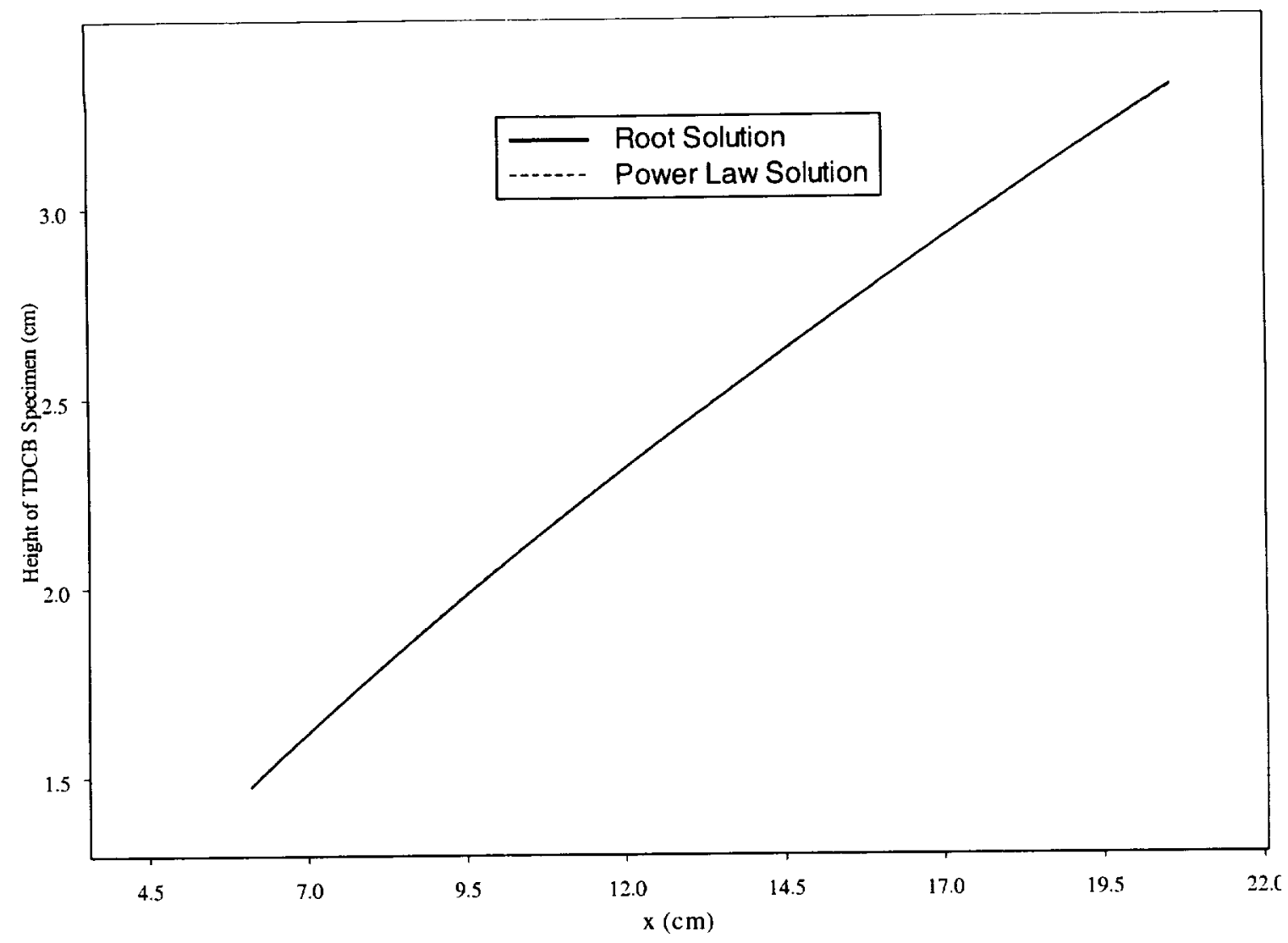

Figure 8: Power law and numerical solution for the root of $m$ versus beam position. 


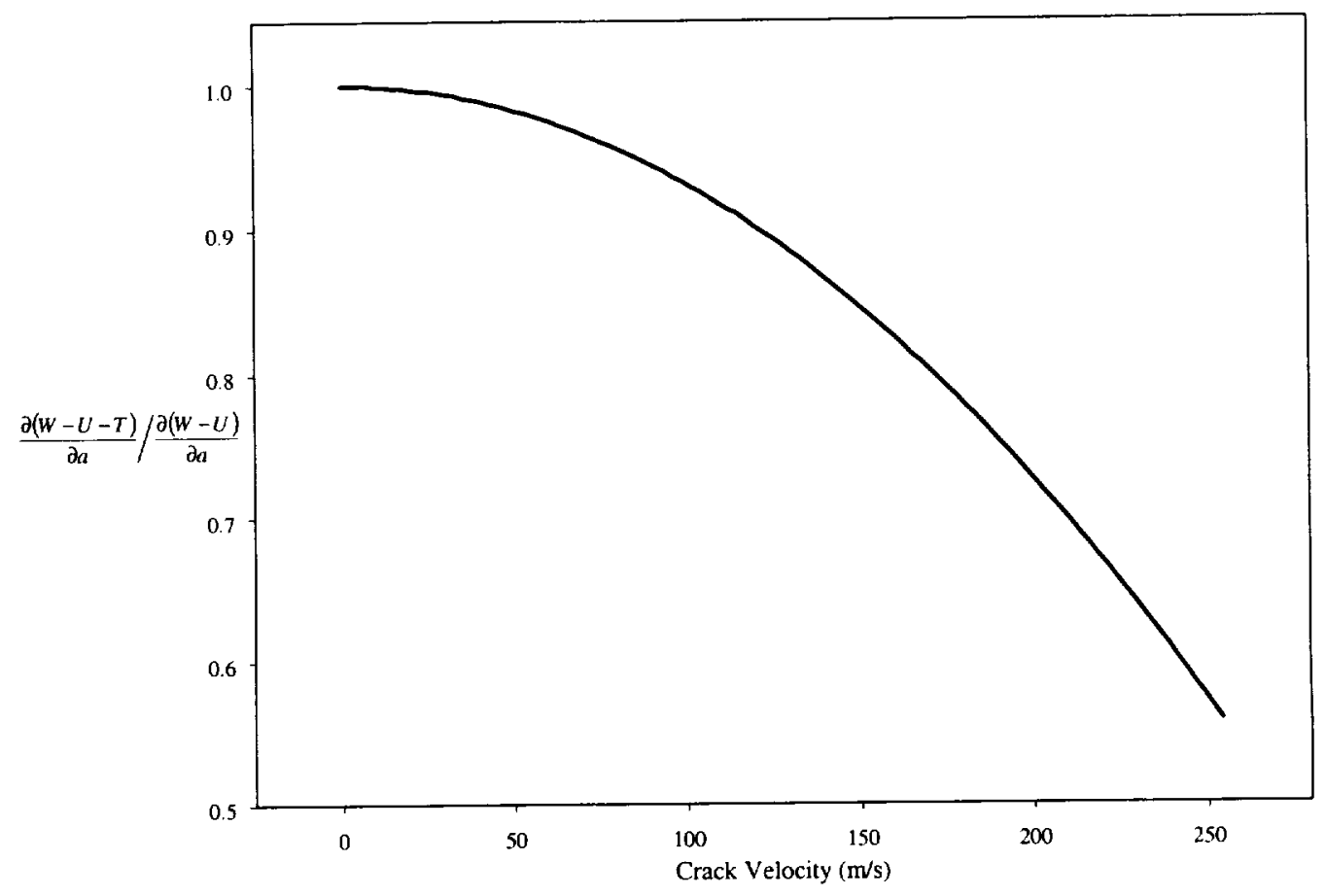

Figure 9: Plot of contribution from kinetic energy versus crack velocity. 


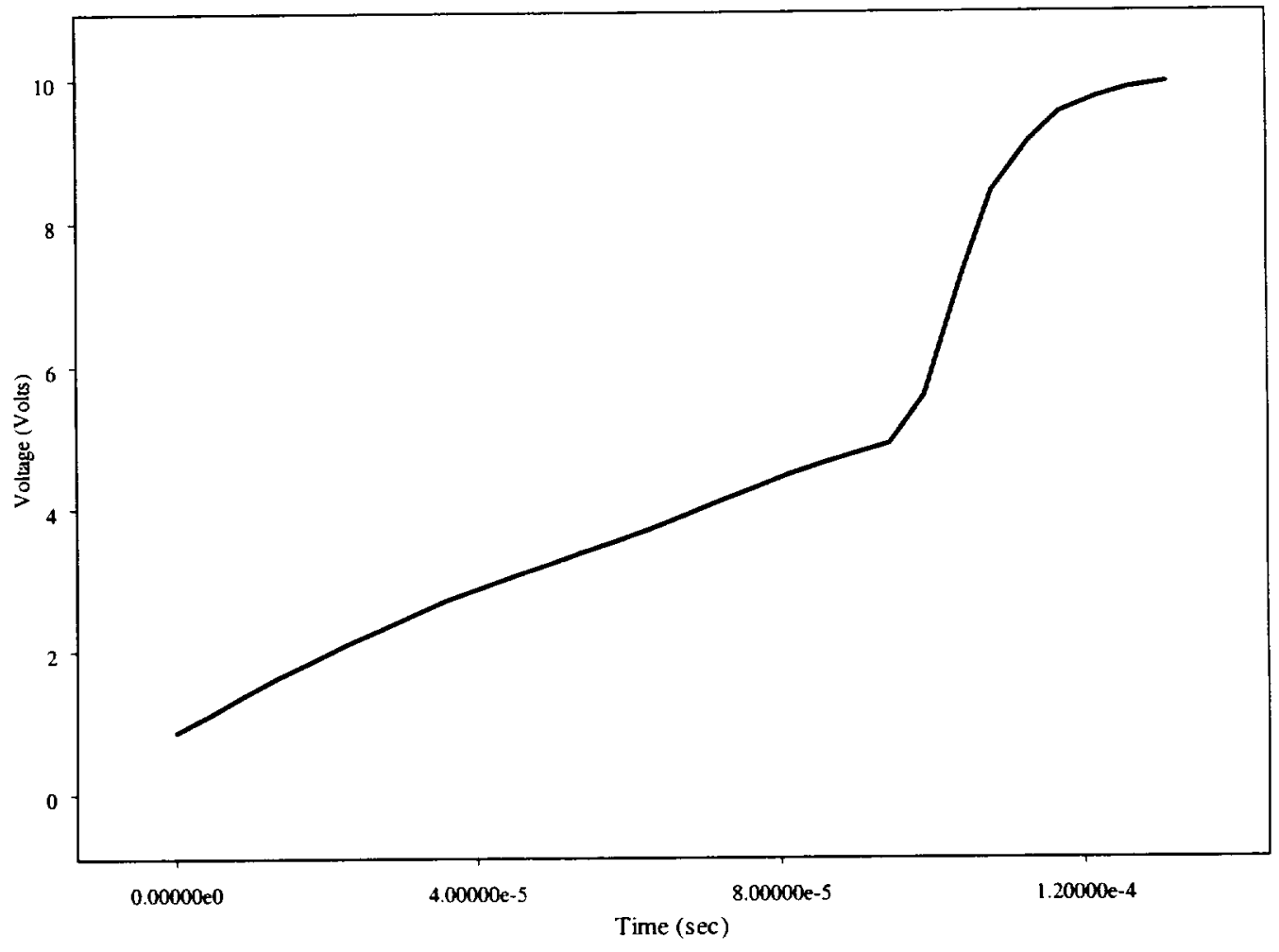

Figure 10: Plot of voltage versus time for crack propagation gauges. 


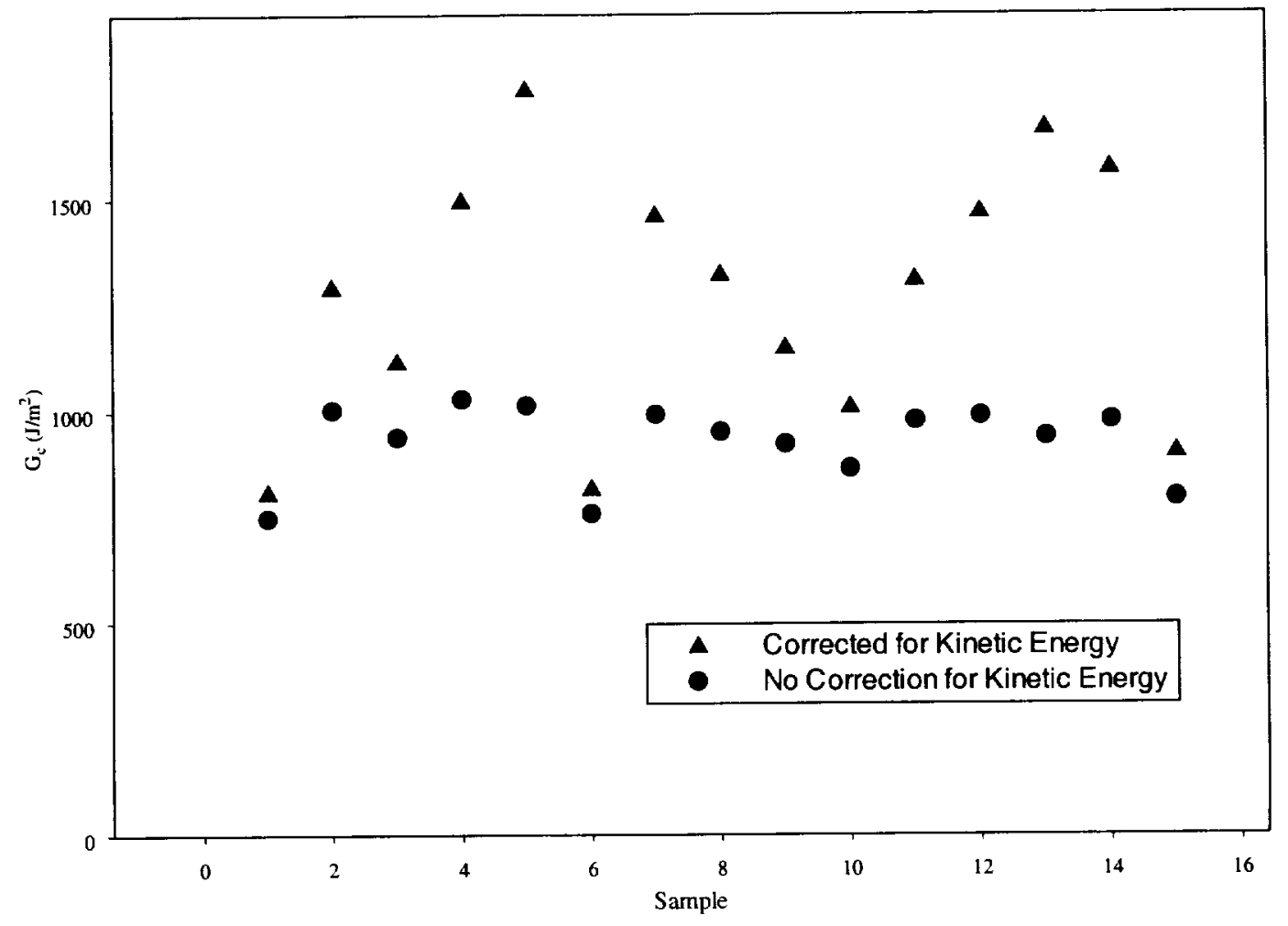

Figure 11: Critical strain energy release rate 